\title{
Essai de description biologique des interactions entre eau de surface et eau souterraine : vulnérabilité d'un aquifère à la pollution d'un fleuve
}

\author{
M. Lafont 1 \\ A. Durbec ${ }^{2}$
}

Mots clés : Rhône, relations nappe-cours d'eau, hydraulique, pollution, descripteurs biologiques, oligochètes.

Le site de l'Ile du Grand Gravier constitue un champ captant pour l'alimentation en eau potable de 80000 équivalenthabitants. Sa situation dans le fleuve Rhône, à l'aval immédiat de l'agglomération lyonnaise et du couloir de la chimie, pose le problème de la vulnérabilité de la ressource en eau à une pollution du fleuve. Le site a fait l'objet de recherches hydrauliques et biologiques au niveau d'une parcelle expérimentale.

L'étude des oligochètes a permis de dresser le diagnostic d'un état de pollution important du fleuve sur le secteur envisagé. Il apparaît de plus que certains taxons d'oligochètes, en particulier le genre Phallodrilus, décrivent une augmentation des échanges nappe-cours d'eau.

L'ensemble de ces données souligne la vulnérabilité du site à une pollution transitant par le Rhône.

Les résultats de cette première approche sont discutés. Il s'avère notamment que les aspects saisonniers n'ont pas été pris en considération. Le développement de la méthodologie est envisagé par l'étude complète de plusieurs autres parcelles.

Contribution to the biological description of relations between surface and underground waters : vulnerability of aquifers to river pollution.

Keywords: Rhône, surface water, groundwater, hydrodynamics, pollution, biological deseribers, Oligochaeta.

The aim of the study is to define the vulnerability of phreatic waters to surface water pollution in a strongly polluted site of the river Rhône. Special emphasis is given to the description by oligochaete communities of the amplification of water exchanges between surface and underground waters, as well as of the degree of pollution at the site. The coarse sediments oligochaete index shows low values that attest to a heavy load of industrial pollution. The absolute and relative abundances of such taxa as Phallodrilus sp. increase in superficial coarse substrata in relation to hydraulic measurements of hydric exchanges amplification. These data emphasize the vulnerability of phreatic waters to pollution by superficial waters. The results are discussed. Seasonal effect was not considered here, and a new study is planned that includes seasonal factors and data on the biology of subterranean waters.

\section{Introduction}

L'impact écologique d'aménagements tels que des barrages ou des extractions de granulats a généralement été étudié sous l'angle des perturbations qu'ils induisent directement sur les biocénoses superficielles ou souterraines (voir par exemple Bou 1977,

1. Division Qualité des eaux, Pêche, Pisciculture. CEMAGREF, Groupement de LYON, 3 Quai Chauveau, F-69336 Lyon Cédex 09.

2. Division Hydrologie - Hydraulique, CEMAGREF, Groupernent de LYON, 3 Quai Chauveau, F-69336 Lyon Cédex 09.
Souchon et al. 1989). Cet impact peut être qualifié d'aigu, dans la mesure oủ les organismes sont parfois totalement éliminés du milieu. En revanche, la description par les organismes aquatiques des perturbations provoquées par ces aménagements sur les relations hydrodynamiques entre la nappe phréatique et les eaux superficielles n'a que rarement été abordée (Lafont \& Durbec 1990). Dans ce dernier cas, la problématique est différente : elle consiste à intégrer la biologie pour décrire un dysfonctionnement chronique des relations hydrodynamiques entre la nappe et le système superficiel. En particulier 
la mise en évidence des zones privilégiées d'échanges hydriques, liées à la présence d'un aménagement, constitue une information majeure. Ces zones d'échange apparaissent comme un danger potentiel et mésestimé de contamination de la nappe phréatique par des substances indésirables apportées par des eaux de surface. De plus, on ne maîtrise pas à plus long terme les risques écologiques liés à un accroissement des échanges entre les eaux souterraines et les eaux superficielles.

D'un point de vue pratique, les invertébrés constituent un matériel biologique de choix. En effet, la richesse de la faune souterraine d'invertébrés n'est plus à démontrer, et les concepts écologiques concernant la dynamique de répartition de cette faune ont fait l'objet de nombreux travaux de synthèse (Gibert 1986, Ginet \& Juberthie 1987, Rouch \& Danielopol 1987...). Divers auteurs ont par ailleurs judicieusement souligné que la connaissance de l'écologie des eaux souterraines pouvait apportẹr beaucoup à la compréhension du fonctionnement des écosystèmes superficiels (Bou 1977, Hynes 1983).

Dans ce contexte, tout aménagement affectant sensiblement les échanges hydrodynamiques entre les eaux souterraines et les eaux superficielles devrait en principe se caractériser par la prolifération d'organismes hypogés dans lẹs horizons superficiels (Lafont 1989, Lafont \& Durbec 1990). Les oligochètes, compte tenu de l'importance des eaux souterraines dans la dynamique de leur répartition, semblent constituer un groupe descripteur d'invertébrés particulièrement intéressant à retenir dans ce type de recherche. En effet, beaucoup d'espèces communes dans les eaux de surface se trouvent également dans le milieu souterrain (Strayer \& Bannon-O'Donnel 1988, Lafont 1989). Inversement, des espèces stygobiontes, comme par exemple Dorydrilus michaelseni (Juget \& Dumnicka 1986), peuvent être hébergées par les sédiments superficiels, mais seulement à proximité d'un aménagement (Lafont 1989). La prise en considération des peuplements d'oligochètes permet également de disposer d'un outil fiable pour apprécier l'impact de rejets polluants, ce qui donnerait la possibilité de bénéficier, sur le même site, d'informations concernant l'état de pollution du milieu et la nature des perturbations hydrauliques.

Cependant, l'état des connaissances donne aujourd'hui une avance certaine aux modèles hydrauliques.
L'objectif de cette recherche a donc consisté à tenter de décrire, d'un point de vue biologique et hydraulique, les potentialités d'échanges hydrodynamiques entre un secteur de cours d'eau, pollué et perturbé au plan hydraulique, et sa nappe d'accompagnement, les retombées pratiques immédiates devant permettre d'apprécier la vulnérabilité potentielle de celle-ci à tout apport polluant.

Les mesures hydrauliques et les relevés biologiques (oligochètes) ont donc été effectués en vue de préciser les deux points suivants :

- appréciation du degré de pollution du secteur, donc des risques potentiels de contamination de la nappe ;

- cartographie hydraulique et biologique des zones d'échanges actifs.

\section{Site étudié}

Le travail a été conduit sur un secteur du Rhône situé à $25 \mathrm{~km}$ en aval de Lyon, l'Ile du Grand Gravier (fig. 1).

Le problème posé par le Rhône dans ce secteur est lié à l'alimentation en eau potable des collectivités environnantes. Compte tenu des pompages effectués dans la nappe $\left(20000 \mathrm{~m}^{3} /\right.$ jour et $80000 \mathrm{~m}^{3} / \mathrm{j}$ maximum autorisé), les échanges hydrodynamiques entre celle-ci et le fleuve sont très perturbés, le Rhône alimentant pratiquement en permanence la nappe phréatique. En conséquence, celle-ci prèsente le risque d'être contaminée par des substances polluantes amenées par le fleuve.

En effet, le site de captage se trouve à l'aval du " couloir de la chimie " qui connaît presque chaque année une pollution accidentelle de premier ordre, rappelant au public la vulnérabilité de la ressource en eau.

Des recherches en cours sur l'effet filtre des berges du Rhône permettent d'acquérir une très bonne connaissance du contexte hydrogéologique et de décrire finement les phénomènes hydrodynamiques (Durbec \& Leduc 1990, C.E.N.G. 1990, B.R.G.M. sous presse).

Les caractétistiques hydrodynamiques de l'aquifère (perméabilité, porosité, drainance par le lit du Rhône, etc) ont été évaluées par différentes méthodes (Durbec \& Leduc 1988) produisant des résultats de valeur régionale et locale : 


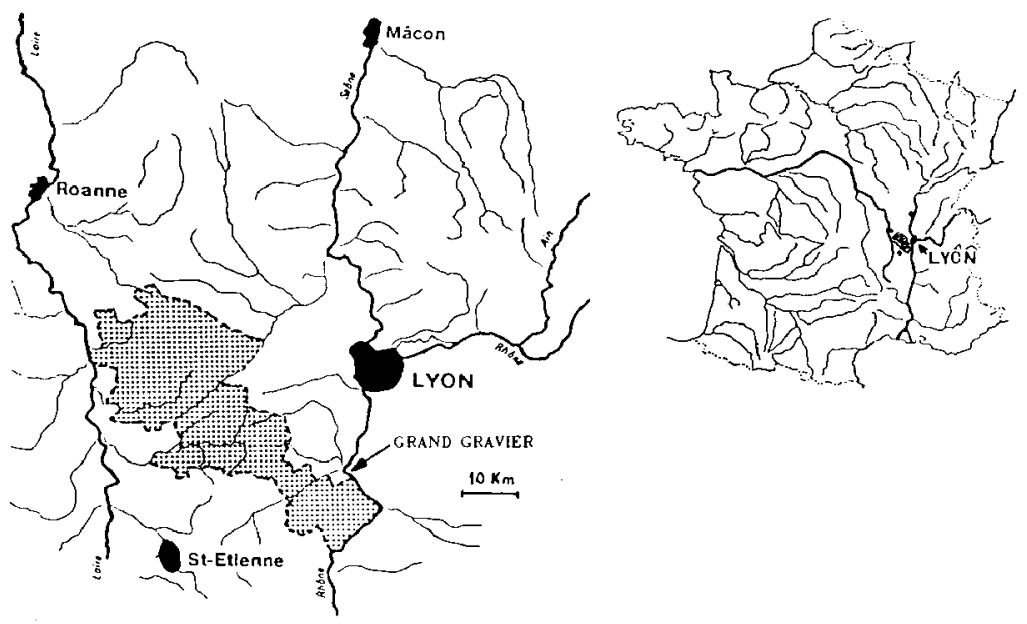

Fig. 1: Localisation de l'lle du Grand Gravier ; en pointillé, zone de distribution d'eau potable concernée.

- en période d'étiage, les captages sont alimentés par le Rhône pour $82 \%$ des volumes extraits;

- les phases transitoires ont montré que le réservoir aquifère de l'Ile du Grand Gravier réagit très rapidement à toute sollicitation du Rhône. Cette réaction s"atténue vers le nord.

Ces indications permettent de proposer une sectorisation hydrodynamique de l'Jle, mais elles ne sont pas suffisantes pour interpréter finement les processus biologiques. Pour cette raison, une parcelle expérimentale a été choisie au nord et un modèle local a été mis au point (fig. 2).

\section{Matériel et méthodes}

Les relevés biologiques ont été réalisés au cours d'une campagne d'échantillonnage en septembre $1989(6 / 9 / 1989)$ dans les sédiments grossiers (blocs, galets, graviers) en courant lent ( $v \leqslant 10 \mathrm{~cm} / \mathrm{s}$ ), sous une épaisseur d'eau d'environ $0,40 \mathrm{~m}$. Quatre échantillons ont été effectués selon un transect longitudinal, à raison d'un échantillon tous les trente mètres.

Chaque échantillon était constitué de deux prélèvements, l'un effectué à l'aide d'un filet dérivé du type Surber $\left(400 \mathrm{~cm}^{2}\right.$ d'ouverture, maille du filet de $0.160 \mathrm{~mm}$, cf. Juget \& Lafont 1982), l'autre à l'aide d'un carottier métallique cylindrique, enfoncé dans le substrat à l'aide d'une masse ( $10 \mathrm{~cm}$ de diamètre, $78,5 \mathrm{~cm}^{2}$ d'ouverture), fermé à l'aide de la paume de la main, (Durbec 1986). Dans les deux cas, une couche de substrat de $10 \mathrm{~cm}$ d'épaisseur est ramassée. Les prélèvements sont fixés sur le terrain au formaldéhyde pour obtenir une solution de 5-6\% de formaldéhyde dans le bocal de récupération. Au laboratoire, les échantillons subissent une lévigation pour séparer la fraction organique de la fraction minérale et sont lavés à l'eau sur un tamis de $0,160 \mathrm{~mm}$ de vide de maille. Le refus de tamis est réparti dans des cuves quadrillées puis homogénéisé. Un sous-échantillonnage est éventuellement effectué en triant à la loupe binoculaire le contenu de $\mathbf{n}$ carrés choisis au hasard pour obtenir un lot de 100 oligochètes, qui sont ensuite montés entre lame et lamelle et examinés au microscope.

Les paramètres hydrodynamiques de l'aquifère ont été déterminés par les méthodes classiques suivantes: 


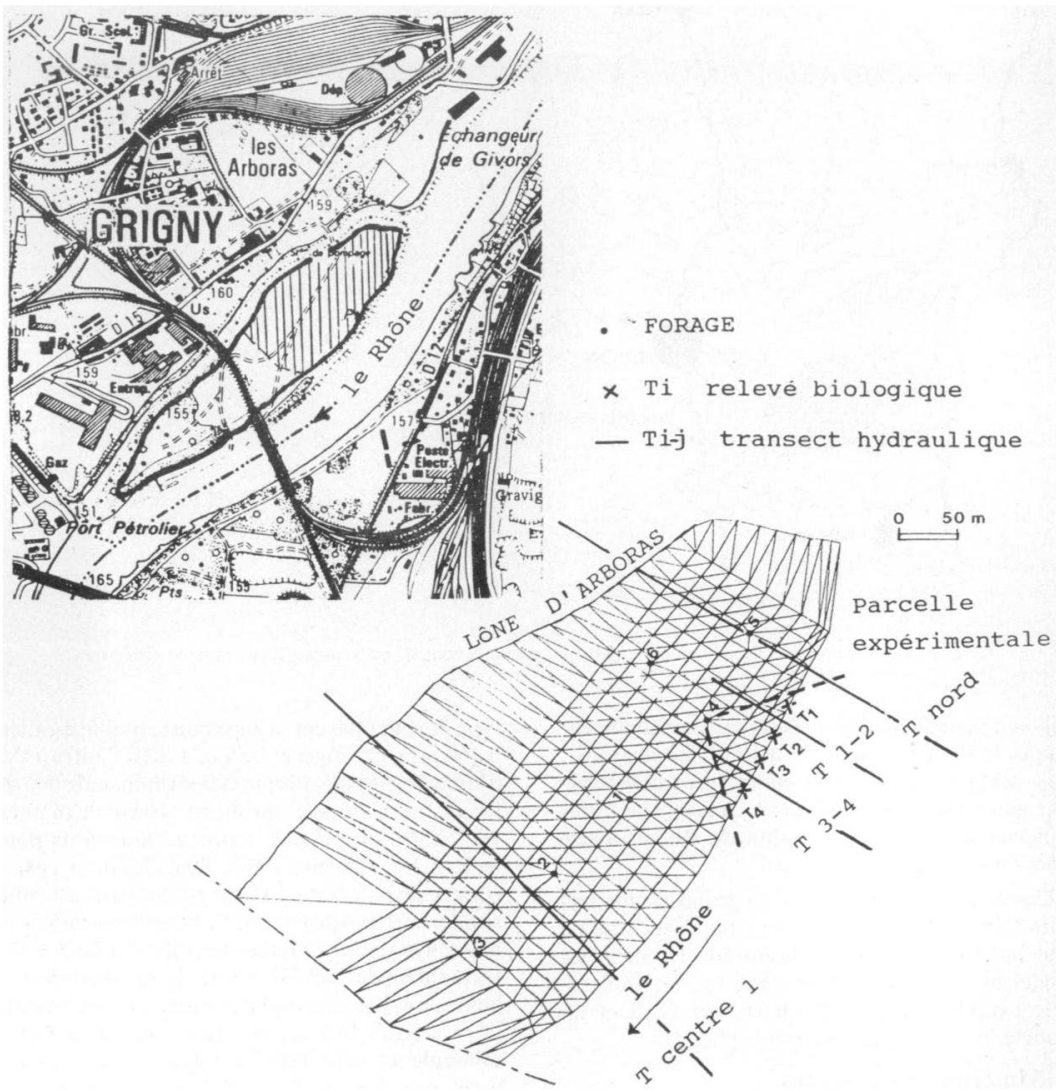

Fig. 2 : Secteur du Grand Gravier : Extrait du maillage spatial, localisation de la parcelle expérimentale, des transects hydrauliques et des relevés biologiques.

- interprétation d'essais de pompage (souvent délicate à cause des très faibles rabattements observés) ;
- corrélation entre propriétés électriques et caractéristiques hydrauliques d'après des campagnes de prospection géophysique électrique ; 
- partition de l'aquifère en zones homogènes en fonction des fluctuations de la surface piézométrique.

Un réseau piézométrique de 20 points, dont 5 sont équipés d'enregistreurs en continu, est suivi depuis 1987 avec une fréquence mensuelle. Les données concernant les niveaux du Rhône nous sont transmises par la Compagnie Nationale du Rhône, le fonctionnement des puits de captage nous est communiqué par la compagnie fermière qui en assure l'exploitation.

Le dépouillement des mesures de terrain a eu pour objectif d'évaluer les échanges entre le Rhône et la nappe. Les limnigrammes enregistrés permettent d'examiner analytiquement les fluctuations du niveau du cours d'eau et leurs répercussions sur l'aquifère en fonction du temps et de la distance à la berge.

A l'échelle du domaine étudié, la modélisation numérique des écoulements souterrains permet d'extrapoler les caractéristiques de la nappe, de simuler différentes situations, de calculer les vitesses de circulation et de quantifier les échanges après avoir étalonné les calculs par référence aux campagnes de mesure. Pour valoriser la grande quantité d'informations disponibles, un modèle prenant en compte l'Ile du Grand Gravier (70 ha) permet une description des échanges avec une résolution fine (fig. 2). Ce modèle de nappe est conceptuel, déterministe et basé sur l'application des lois fondamentales de la mécanique des fluides à l'écoulement de l'eau en milicu poreux (loi de Darcy, hypothèse de Dupuit).

\section{Résultats}

\subsection{Caractéristiques hydrodynamiques}

\subsubsection{ECHELLE REGIONALE}

Différentes simulations ont permis de caractériser le fonctionnement actuel et d'apprécier l'impact de divers aménagements sur la nappe. Ont été testés, en régime permanent puis transitoire :

- l'équipement de nouveaux captages sur l'Ile du Grand Gravier dans différentes conditions d'exploitation ;

- le curage de la lône d'Arboras en vue de sa mise en eau permanente.

L'étalonnage du modèle a été réalisé sur la base des campagnes de 1987 et 1988 . Le régime permanent était établi courant septembre 1987, une campagne générale ayant eu lieu le $29 / 09 / 87$. Ont été pris en compte :

- l'analyse comparée des niveaux de la nappe et des cours d'eau (Rhône et Garon) ;

- les essais de pompage près de limites à potentiel constant.

L'influence prépondérante, et rapide, du Rhône sur toute la bordure sud-est de l'aquifère est évidente. Dans la vallée du Garon, les échanges sont limités par le tarissement de la rivière pendant les mois d'été, la faible largeur et le colmatage du lit.

\section{Origine de l'eau pompée}

L'eau en provenance directe du Rhône au travers des berges de l'tle (y compris de la lône si celle-ci est remise en eau) varie de $75 \%$ (état actuel) à $95 \%$. La part du Rhône est donc essentielle.

Les apports du Rhône vers la nappe à l'amont de l'lle ne dépassent jamais $10 \%$. Ils sont repris en totalité ou en partie par les pompages du Grand Gravier après un transit plus ou moins long dans l'aquifère.

La part de la « nappe du Garon », en bordure sud-ouest, dans l'alimentation du Syndicat est donc réduite. Sa proportion diminue au fur et à mesure que les prélèvements augmentent.

\section{Sectorisation des échanges Rhône-nappe}

La relation Rhône-nappe peut être quantifiée en régime transitoire de façon à définir les conditions de réalimentation du sous-sol de l'Ile du Grand Gravier, lors d'une crue du Rhône.

La période retenue pour l'évaluation des volumes échangés est celle du 24/09/87 au 03/10/87. Le premier jour correspond à la fin du régime permanent, avant une crue du Rhône.

Les résultats du modèle numérique sont analysés sur quatre transects perpendiculaires au fleuve, répartis du nord au sud de l'lle (Nord, Centre $n^{0}$ 1 , Centre $n^{\circ} 2$, Sud). La propagation dans le milieu poreux souterrain de l'onde de crue s'accompagne d'un déphasage fonction de la transmissivité, de l'emmagasinement des alluvions, du colmatage de la berge, et bien sûr de la distance au cours d'eau.

A titre d'exemple, la figure 3 illustre le cas du transect nord, jusqu'à 850 mètres du fleuve. Les échanges sont de plus en plus intenses en allant vers le sud de l'ile. En examinant, entre deux pas de temps 


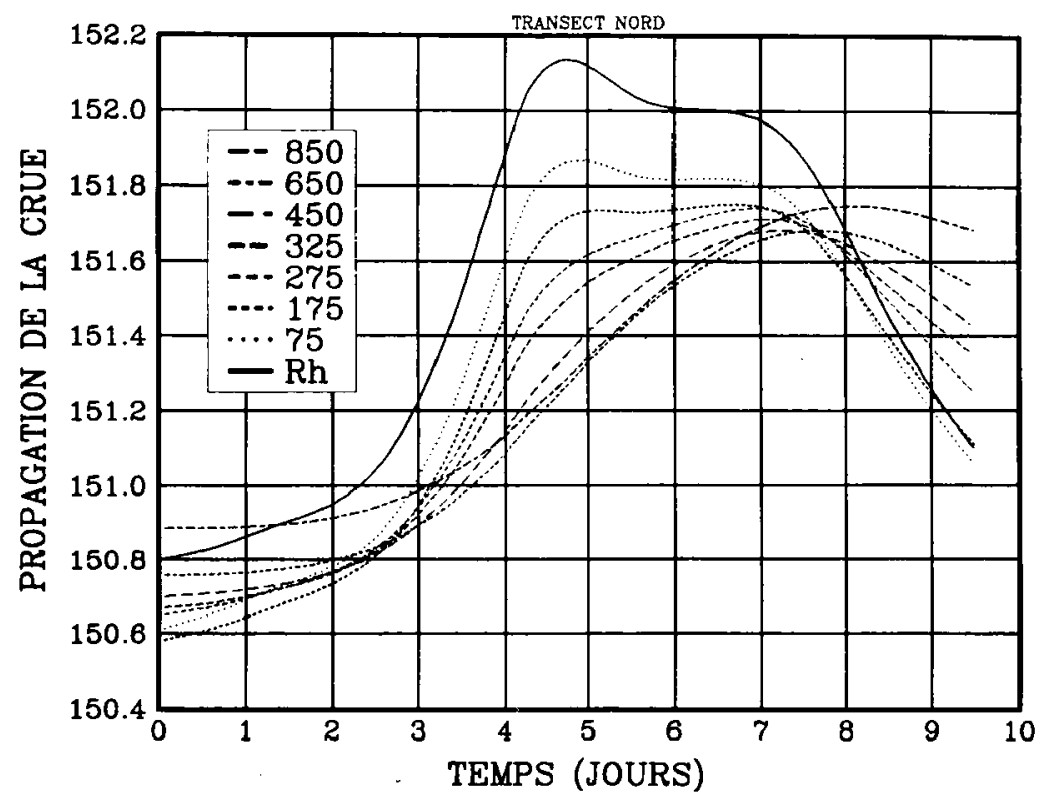

Fig. 3 : Propagation danș là nappe en fonction du temps d'une crue du Rhône (cotes NGF : $150,4-150,2)$ à différentes distances de la berge ( 75 à 850 mètres); cas du transect nord.

successifs, l'évolution des volumes stockés ou déstockés sur un transect (Ubell 1987), on obtient l'j]lustration de la nervosité ou de l'inertie de la nappe du Rhône sollicitée par une modification du niveau du cours d'eau (fig. 3).

Il résulte de cette approche que la ressource en eau captée est vulnérable à une pollution transitant par le Rhône et ce d'autant plus que les captages se situeront au sud de l'lle du Grand Gravier. Nous avons choisi de faire porter nos efforts de recherche sur la partie nord, dans un premier temps.

\subsubsection{EChELle LOCALE - PARCELLE NORD DE L'ILE}

Pour une étude précise des vitesses de circulation visant à une sectorisation à l'échelle décamétrique, la discrétisation de l'espace doit être assez fine.
Le modèle utilise des mailles triangulaires dont les plus petites ont une surface de $30 \mathrm{~m}^{2}$. La plus grande précision a été apportée autour des captages et sur la parcelle expérimentale au nord de l'Ile.

Les lieux d'échantillonnage d'oligochètes (T1 à T4) ont servi de points de repère pour deux nouveaux transects perpendiculaires au Rhône, distants de quarante mètres environ. Sur chacun des transects la simulation de la crue du $24 / 09$ au 03/10/87 a permis de caractériser les échanges à l'échelle de la parcelle, suivant la méthode décrite en 4.1.1. Les vitesses réelles calculées sont significativement plus importantes entre T3 et T4 qu'entre $\mathrm{T} 1$ et $\mathrm{T} 2$, de l'ordre de 10 mètres par jour (fig. 4). Ceci traduit que le milieu est, à gradient constant, plus perméable 


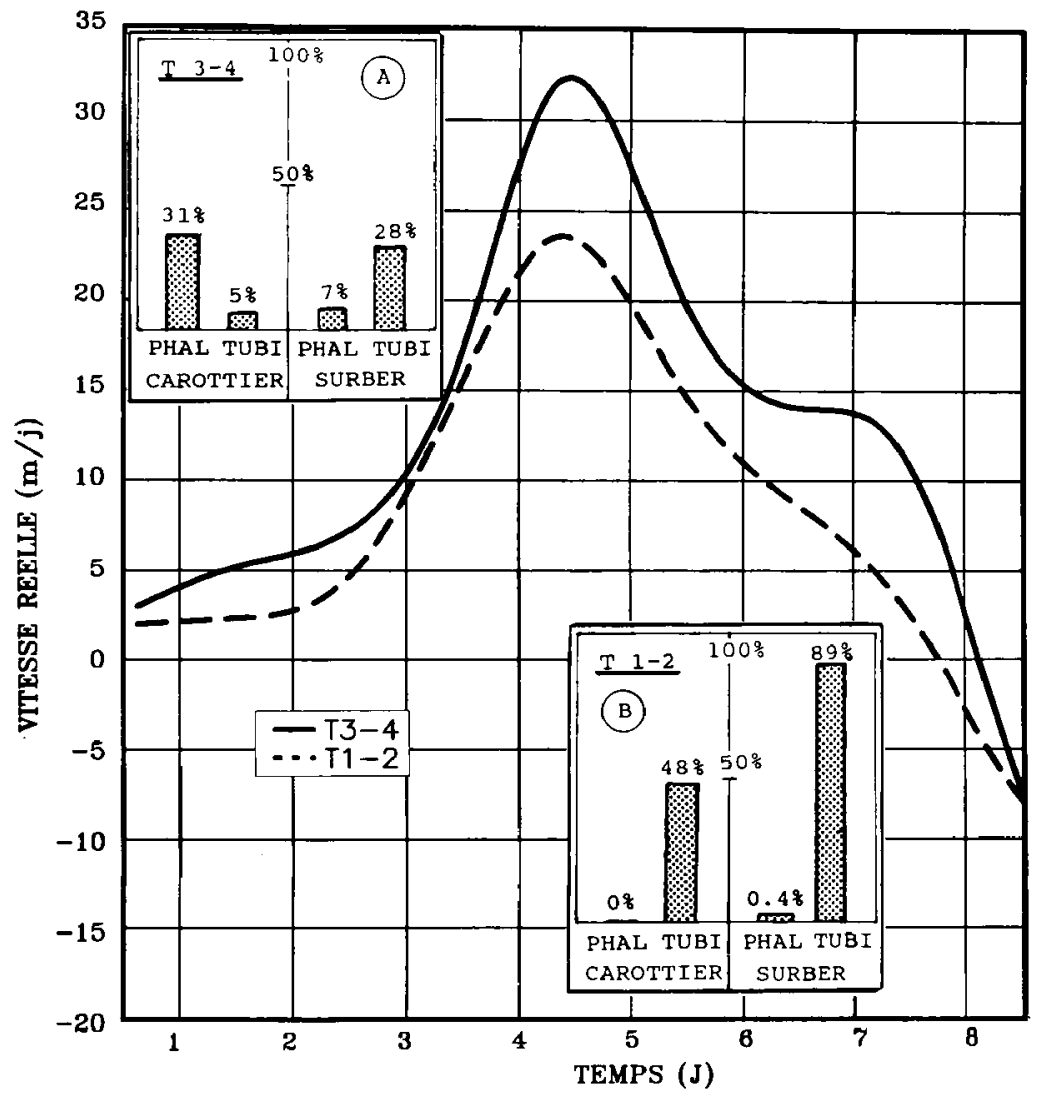

Fig. 4 : Relation entre les peuplements d'oligochètes et l'évolution de la vitesse réelle d'écoulement dans les alluvions grossières rivulaires (Transects T1.2 et T3-4) : A et B : Abondance relative des Tubificidae sauf Phallodrilus (TUBI) et du genre Phallodrilus (PHAL) dans les prélèvements effectués au carottier et au filet surber. 
dans la partie sud de cette parcelle. La confirmation de ce résultat pourra être apportée par infiltrométrie directe, à condition de veiller à s'affranchir des hétérogénéités en multipliant les lieux de mesures.

\subsection{Résultats concernant les oligochètes}

Trente trois espèces ou taxons ont été recensés au total (Tableau I). Le Tubificidac Phallodrilus riparius, décrit dans le Tambre en Espagne (Giani \& Martinez-Ansemil 1981), est nouveau pour le bassin du Rhône.

\section{Caractérisation de l'impact des rejets industriels}

L'impact des rejets industriels a été estimé à partir des quatre prélèvements effectués au filet de type Surber (Tableau II), afin de se conformer au protocole d'échantillonnage préconisé pour le calcul de l'indice oligochètes de pollution « sédiments grossiers " (IOSG), décrit dans un travail précédent (Lafont 1989). Cet indice, qui varie de I (pollution industrielle très importante) à 10 (bonne qualité du milieu), est basé sur la prise en considération du pourcentage de Tubificidae découpé en 10 classes. L'absence de pollution est décrite par un pourcentage de Tubificidae $\leqslant 1 \%_{0}(I O S G=10)$, un état accusé de pollution industrielle est décrit par un pourcentage $\geqslant 70 \%($ IOSG $=1)$. Dans le cas du Grand Gravier, la valeur moyenne de l'indice IOSG atteint 3/10 (Tableau II).

L'impact des rejets industriels se révèle très important sur le secteur étudié. De plus, les effectifs moyens d'oligochètes s'avèrent relativement peu élevés ( 784 spécimens $/ 0,1 \mathrm{~m}^{2}$ ) par référence à ceux que I'on peut observer dans des sédiments grossiers pollués organiquement $\left(>10000\right.$ spécimens $/ 0,1 \mathrm{~m}^{2}$, Lafont 1989). Cette dernière observation permettrait de suspecter la présence de substances à caractère toxique.

\section{Description des relations hydrodynamiques entre la nappe et le cours d'eau}

On observe, du point $\mathrm{T} 1$ au point $\mathrm{T} 4$, une augmentation du pourcentage de taxons inféodés au milieu interstitiel et considérés a priori comme des descripteurs de relations étroites entre la nappe et les eaux superficielles (Tableau II), en particulier le genre Phallodrilus. Deux zones distinctes peuvent être individualisées :

- les points $\mathrm{T} 1$ et $\mathrm{T} 2$, où domine l'impact de la pollution, caractérisés par des pourcentages élevés de Tubificidae $(34,8$ à $92,9 \%$, Tableau II) ;
- les points T3 et T4, où le pourcentage de Tubificidae diminue en même temps qu'augmente la proportion des Enchytraeidae et des genres Phallodrilus, Cernosvitoviella, Pristina et Pristinella. Cette tendance semblerait confirmée par l'examen de relevés de faune souterraine, effectués en avril 1990 dans le sous-écoulement du fleuve sur le même secteur (Tableau II). En effet, les pourcentages du genre Phallodrilus se révèlent effectivement plus élevés sur le transect T4. Cet ensemble d'observations concorde avec l'examen des données hydrauliques (fig. 4).

\section{Conclusion - Discussion}

Sur un plan pratique cette première étude apporte des enseignements intéressants. En premier lieu, elle a permis de déboucher sur une cartographie biologique et hydraulique du secteur retenu (fig. 4). Il existe, sur environ $50 \%$ de ce secteur, une zone perméable d'échanges actifs, dont la présence simultanée avec un syndrome important de pollution du Rhône, laisse présager une certaine vulnérabilité de la nappe à cet endroit.

En second lieu, les méthodologies utilisées jusqu'ici en hydraulique pour quantifier les échanges hydrodynamiques entre un cours d'eau et sa nappe d'accompagnement font appel à des protocoles expérimentaux lourds et coûteux. Leur mise en œuvre a souvent lieu après un choix d'implantation subjectif qui n'est jamais optimal. S'il ne s'agit pas de remplacer l'hydraulique par la biologie, il peut s'avérer fort utile de faire appel à cette dernière pour effectuer une sectorisation préliminaire à l'équipement de sites d'observation. Dans le cas du Grand Gravier, il serait souhaitable d'implanter un site d'observation plus en aval, sur le point 4 . Cette remarque ne remet pas en cause les présents résultats mais doit être prise en considération pour l'implantation de sites futurs.

Sur un plan plus fondamental, les résultats posent de nombreux problèmes méthodologiques et conceptuels.

En ce qui concerne l'échantillonnage, on constate que les oligochètes de petite taille (Naididae) et inféodés au sous-écoulement (genre Phallodrilus, Tableau I et II) se révèlent significativement mieux représentés dans les prélèvements effectués au carottier. En revanche, les Tubificade (autres que Phallodrilus, Tableau II), en particulier les spécimens adultes de grande taille, sont plus abondants dans les prélèvements effectués 
Tableau I : Liste des espéces d'oligochètes récoltées sur les 4 transects (Tl à T4) de l'ile du Grand Gravier ; SU. = filer surber ; CA. = carotier.

\begin{tabular}{|c|c|c|c|c|c|c|c|c|}
\hline & SU & $\begin{array}{l}1 \\
\mathrm{CA} \text {. }\end{array}$ & SU. & $\begin{array}{l}2 \\
C A\end{array}$ & SU. & $\begin{array}{l}3 \\
\mathrm{CA} \text {. }\end{array}$ & SU. & CA. \\
\hline $\begin{array}{l}\text { Lumbriculidae } \\
\text { Stylodrilus sp. } \\
\text { Tubificidae }\end{array}$ & 1,9 & & 4.7 & 2,1 & 2,6 & 8,4 & 10,1 & 1 \\
\hline Immatures avec soies capilaires & 19,8 & 7,6 & 24,4 & 10,4 & 3,6 & & & 1 \\
\hline $\begin{array}{l}\text { Immatures sans soies capillaires } \\
\text { Tubifex tubifex }\end{array}$ & $\begin{array}{r}36 \\
4,1\end{array}$ & $\begin{array}{r}12,1 \\
1,5\end{array}$ & 20,5 & 25 & 20,7 & 0,9 & 18.8 & 5.1 \\
\hline T. kessleri & & & 0,8 & & & & & \\
\hline $\begin{array}{l}\text { Limmodrilus hof fmeister } \mathrm{i} \\
\text { L. claparedeanus }\end{array}$ & 16,9 & $\begin{array}{l}9,1 \\
1,5\end{array}$ & $\begin{array}{r}31,4 \\
0,8\end{array}$ & 16,7 & 5,5 & & 2.9 & \\
\hline $\begin{array}{l}\text { Psammoryctides barbatus } \\
\text { Potamothrix nammoniensis }\end{array}$ & 9 & & $\begin{array}{l}3,1 \\
0,8\end{array}$ & & 1 & & & \\
\hline P. heuscheri & 7,1 & 1,5 & $\begin{array}{l}0.8 \\
0.8\end{array}$ & 2,1 & & 0,9 & & \\
\hline Spirosperma velutinus & & 1.5 & & & & & & 1 \\
\hline $\begin{array}{l}\text { Aulodrilus limnobius } \\
\text { A. pigueti } \\
\text { Branchiura sowerbyi }\end{array}$ & & 1,5 & $\begin{array}{l}0,8 \\
0,8\end{array}$ & $\begin{array}{l}4,2 \\
2,1\end{array}$ & 1 & 0,9 & $\begin{array}{l}1,5 \\
1,5\end{array}$ & $\bar{i}$ \\
\hline Phallodrilus (immatures) & & & 0,8 & & & 14 & 14,5 & 40,8 \\
\hline$\frac{\text { Phallodrilus riparius }}{\text { Naididae }}$ & & & & & & $4, ?$ & & 2 \\
\hline Chaetogaster diaphanus & & 4,5 & & 2,1 & 3,6 & 8,4 & 2,9 & \\
\hline $\begin{array}{l}\text { C. diastrophus } \\
\text { Nais barbata }\end{array}$ & & & 3,9 & $\begin{array}{l}2,1 \\
2,1\end{array}$ & 1,9 & & 2,9 & 2 \\
\hline N. bretscher $i$ & 1,1 & 13,6 & 0,8 & & 11,6 & 9,3 & & 4,1 \\
\hline N. christinae & & & & & 1 & & & 1 \\
\hline N. communis & 1,1 & & & 2,1 & 1 & & 1,5 & \\
\hline N. pardalis & 1,9 & 32 & 2.4 & 25 & 33,3 & 31,8 & 30,4 & 14,3 \\
\hline Stylaria lacustris & 1,1 & 12,1 & 1,6 & 2,1 & 6,1 & 0,9 & 1,5 & \\
\hline Veldovskyella intermedia & & & 0.8 & & & & & \\
\hline$\frac{\text { Pristinella osborni }}{\text { Pristina aequiseta }}$ (foreli) & & 1.5 & & & & $\begin{array}{l}0,9 \\
7,5\end{array}$ & 2,9 & 3,1 \\
\hline $\begin{array}{l}\text { Lumbricidae g. sp. } \\
\text { Enchytraeidae }\end{array}$ & & & & & & 2,8 & & \\
\hline Enchytraeidae g. $\mathbf{s p}$. & & & & 2,1 & & & 1,5 & 2 \\
\hline Marionina argentea & & & & & 3,6 & 1,9 & 1.5 & 3,1 \\
\hline Lumbricillus Sp. & & & & & 2.6 & 3,7 & 2.9 & 1 \\
\hline Cernosvitoviella sp. & & & & & 1 & 2,8 & 2,9 & 17,3 \\
\hline Nombre total $/ 400 \mathrm{~cm}^{2}$ (Surbers) & 367 & & 508 & & 309 & & 69 & \\
\hline Nombre total $/ 78,5 \mathrm{~cm}^{2}$ (carottes) & & 66 & & 48 & & 107 & & 98 \\
\hline Nombre total $/ 0,1 \mathrm{~m}^{2}$ & 918 & $84 \uparrow$ & 1270 & 611 & 773 & 1363 & 173 & 1248 \\
\hline Nombre d'especes & 9 & 11 & 15 & 13 & 15 & 15 & 15 & 15 \\
\hline
\end{tabular}


Tableau II : Abondance relative d'espèces ou de taxons particuliers d'oligochètes dans le Grand Gravier : SU. = prélèvements effectués au filet ; $\mathrm{CA} .=$ prélèvements effectués au carottier ; $B R=$ prélèvements effectués à la pompe Bou-Rouch (à - 0,5 $\mathrm{m}$ et $1 \mathrm{~m}$ de profondeur dans le substrat ; relevés réalisés en avril 1990).

* espèces ou taxons considérés comme descripteurs de relations étroites entre la nappe et le cours d'eau (Lafont, 1989).

\begin{tabular}{|c|c|c|c|c|c|c|c|c|c|c|}
\hline $\begin{array}{l}\text { Points d'échantillonnage } \\
\text { Appareil utilise }\end{array}$ & $:$ SU. & $\begin{array}{l}1 \\
\text { CA. } \%\end{array}$ & su. $\%$ & $\stackrel{2}{C A} .2$ & $\mathrm{Su}^{\mathrm{T}}$ & $\begin{array}{l}3 \\
C A\end{array}$ & $\mathrm{su}^{\mathrm{T}}$ & $\stackrel{4}{C A}$. & ${ }_{B R}^{T}{ }^{T}$ & $\mathrm{TR}^{\mathrm{T}}{ }^{4}$ \\
\hline \multicolumn{11}{|l|}{ Tubificidae } \\
\hline (excepté S. velutinus) & 92,9 & 34,8 & 85,8 & 60.5 & $3 \uparrow, 8$ & 21,4 & 39,2 & 49,9 & 58,6 & 86,4 \\
\hline Naididae & 5,2 & 53.7 & 9.5 & 35,5 & 58,5 & 58,8 & 42,1 & 24,4 & - & 4.5 \\
\hline Lumbriculidae & 1,9 & - & 4,7 & 2,1 & 2,6 & 8,4 & 10,1 & 1 & - & - \\
\hline Enchytraeidae* & - & - & - & 2,1 & 7,2 & 8,4 & 8,8 & 23,4 & 41,4 & 9,1 \\
\hline Incice IOSG $/ 10$ & 1 & & 1 & & 5 & & 5 & & & \\
\hline \& Phallodrilus* sp. & - & - & 0,8 & - & - & 18,7 & 14,5 & 42,8 & 3,4 & 40,9 \\
\hline $\begin{array}{l}\text { * Tubificidae } \\
\text { (excepté Phallodrilus sp.) }\end{array}$ & 92,9 & 34,8 & 85 & 60,5 & 31,8 & 2,7 & 24,7 & 7,1 & 55,2 & 45,5 \\
\hline Pristina et Pristinella spp.* & - & 1,5 & - & - & - & B, & 2,9 & 3,1 & - & - \\
\hline Cernosvitoviella sp." & - & - & - & - & 1 & 2,8 & 2.9 & 17,3 & 3,4 & - \\
\hline Spirosperma velutinus* & - & 1,5 & - & - & - & - & - & 1 & - & - \\
\hline
\end{tabular}

au filet Surber, qui peut privilégier les organismes vivant en surface, notamment ceux qui dominent la face inférieure de pierres dont le diamètre est supérieur à celui du carottier utilisé. Ce problème n'est pas insoluble et la mise au point d'un appareil présentant les avantages des deux systèmes est envisagé.

Les résultats sont limités à un seul site d'observation, ce qui ne permet pas de proposer un modèle dans l'immédiat. En particulier, les données saisonnières ne sont pas prises en considération, notamment lorsque le sens des échanges s'inverse (nappe vers cours d'eau).

Cependant, les résultats sont encourageants dans la mesure où l'on peut observer, au moins dans ce cas précis et très ponctuel, une relation hydrauliquebiologie qui confirme les hypothèses émises précédemment. Certes, le jeu de données ne permet pas de discuter du caractère descripteur des espèces avec une validité suffisante. Mais de nombreux faits semblent concordants et corroborer diverses remarques écologiques. En premier lieu, la valeur descriptrice de relations hydrodynamiques affectée au genre Phallodrilus (Lafont 1989) semble bien ici un fait confirmé par les mesures hydrauliques et s'appliquerait également au genre Cernosvitoviella (Tableau II).

En second lieu, il est envisageable de considérer que l'examen des espèces d'oligochètes permettrait de décrire le sens privilégié des échanges hydriques. Par exemple, la présence de Propappus volki dans les sédiments fluviaux de surface serait liée à un rééquilibrage entre le cours superficiel du Rhône et son sous-écoulement en fin de décrue (Gaschignard 1984a, 1986), donc avec un flux d'échanges dans le sens nappe-cours d'eau. Nous avons nous-même constaté la prolifération dans le Doubs de cette espèce, à l'étiage et à l'aval de sablières entraînant un surcreusement du lit de la rivière (Lafont \& Durbec 1990). La même observation se relate pour les genres Pristina et Pristinella à l'aval de sablières dans la Dordogne et la Dore, ces Naididae étant en général plutôt abondants dans le sous-écoulement des cours d'eau ou le milieu souterrain (Dole 1983, Juget \& Dumnicka 1986). La recolonisation après une crue d'un secteur au lit très instable (canal de Miribel, parallèle au Rhône) s'effectue, pour le Lumbriculidae Stylodrilus heringianus, à partir du sousécoulement (Gaschignard 1984b). On le constate, toutes ces espèces suivent un flux d'échange qui s'effectue dans le sens nappe-cours d'eau à l'inverse du Grand Gravier. Précisément, dans ce dernier secteur, $P$. volki est absente, et les genres Stylodrilus, Pristina et Pristinella sont peu représentés. Ces observations tendraient à suggérer que l'examen des espèces décrirait le sens des échanges entre la nappe et les eaux superficielles. Cependant, d'autres facteurs 
entrent en ligne de compte, comme la saison ou les contaminations industrielles, $P$. volki se révélant une espèce sensible aux rejets industriels (Lafont 1989).

On notera d'ailleurs que les rejets industriels peuvent présenter deux effets simultanés sur les oligochètes, d'une part en éliminant les espèces (effet létal), d'autre part en provoquant une migration de celles-ci dans les alluvions pour échapper aux substances toxiques (Lafont 1989). Cet aspect du problème sera considéré par la suite, dans la mesure où il paraît évident que parmi les espèces décrivant les relations hydriques, on pourra trouver des formes sensibles ou résistantes à la pollution.

Les recherches futures seront axées sur la prise en considération du facteur saisonnier dans le secteur du Grand Gravier, sur la reconnaissance de sites supplémentaires, comme l'aval du Grand Gravier et d'autres champs captants, avec des degrés divers de pollution et de perturbations hydrauliques, en incluant l'étude des eaux souterraines sur un plan hydraulique et biologique, ce dernier aspect ayant seulement été ébauché dans cette étude.

\section{Remerciements}

Les auteurs remercient l'équipe d'Ecologie Souterraine de I'URA CNRS 367 qui a effectué les relevés biologiques dans le sous-écoulement et Madame M. Taillole qui a dactylographié le manuscrit.

\section{Travaux cités}

Bou (C.). 1977. -- Conséquences écologiques de l'extraction des alluvions récentes dans le cours moyen du Tarn. Bull. Ecol., 8 (4) : $435-444$.

B.R.G.M. 1990. - Vulnérabilité des captages de l'île du Grand Gravier face à une pollution du Rhône. Eıude du pouvoir de filtration des berges. Etude sédimentologique détaillée. Rapport SGR Rhöne-Alpes : sous-presse.

C.E.N.G. 1990. - Etude du pouvoir de filtration des berges et de la vulnérabilité des captages de l'île du Grand Gravier (Rhône) face à une pollution du fleuve. Compte rendu des opérations de traceurs effectuées en 1989:110 p.

Dole (M.J). 1983. - Le domaine aquatique souterrain de la plaine alluviale du Rhône à l'est de Lyon. 1 : diversité hydrologique et biocénotique de trois stations représentatives de la dynamique fluviale. Vie et Milieu, $33(3 / 4): 219-229$.

Durbec (A.). 1986. - Sectorisation des berges des ballastières en eaux. Application à l'étude des échanges hydrodynamiques avec la nappe phréatique d'Alsace, au nord de Strasbourg. These de Doctorat, Universite Louis Pasteur, Strasbourg : $202 \mathrm{p}$.

Durbec (A.) \& Leduc (C.). 1988. - Etude hydrogéologique de l'île du Grand Gravier et de la basse vallée du Garon. Rapport final, CEMAGREF, decembre 1988: $253 \mathrm{p}$.
Durbec (A.) \& Leduc (C.). 1990. - Vúnérabilité des captages de l'île du Grand Gravier face à une pollution du Rhone. Etude du pouvoir de filtration des berges. Rapport intermédiaire CEMAGREF, janvier 1990: $39 \mathrm{p}$.

Gaschignard (O.). 1984a. - La granulométrie du sédiment et l'évolution du benthos danś un bras vif du Rhône. Rev. Sci. Eau, 3 : 279-293.

Gaschignard (O.). 1984b. - Impact d'une crue sur les macroinvertébrés benthiques d'un bras du Rhône. Verh. Internat. Verein. Limnol., 22 : 1997-2001.

Gaschignard-Fossati (O.). 1986. - Répartition spatiale des macroinvertèbrés benthiques d'un bras vif du Rhône : rôle des crues et dynamique saisonnière. Thèse de Doctorat, Université Lyon $I: 197$ p. + annexes.

Giani (N.) \& Martinez-Ansemil (E.). 1981. - Observaciones acerca de algunos Tubificidae (Oligochaeta) de la Peninsula Ibérica, con la descripción de Phallodrilus riparius n. sp. Annts Limnol., 17 (3) : 201-209.

Gibert (J.). 1986. - Ecologie d'un système karstique jurassien. Hydrogéologie, dérive animale, transits de matières, dynamique de la population de Niphargus (Crustacé Amphipode). These de Doctorat d'Etat és Sciences, Université Lyon I: $368 \mathrm{p}$.

Ginet (N.) \& Juberthie (C.). 1987. - Le peuplement animal des Karsts de France (éléments de biogéographie souterraine pour les invertébrés). Première partie : la faune aquatique. Karstologia, $10: 43-51$.

Hynes (H.B.N.). 1983. - Groundwater and stream ecology. Hydrobiologia, 100 : 93.99.

Juget (J.) \& Lafont (M.). 1982. - L'échantillonnage de la faune benthique : revue des techniques de prélèvements, d'extraction et de tri; application aux Oligochètes. Rev. Sci. Eau, 1 (3) : 243-254.

Juget (J.) \& Dumnicka (E.). 1986. - Oligochata (incl. Aphanoneura) des eaux souterraines continentales. In Stygofauna mundi, L. Botosaneanu (ed.), E. J. Brill, Leiden : 234-244.

Lafont (M.). 1989. - Contribution à la gestion des eaux continentales : utilisation des oligochètes comme descripteurs de l'état biologique et du degré de pollution des eaux et des sédiments. These de Doctorat d'Etat ès Sciences, UCBL Lyon $l: 311 \mathrm{p} .+$ annexes $92 \mathrm{p}$.

Lafont (M.) \& Durbec (A.). 1990. - Description biologique des relations hydrauliques entre la nappe et les eaux superficielles : Application à l'impact de gravières. Actes du Symposium "Bravo l'Eau", Comar 28-30 mars 1990: 11 p.

Rouch (R.) \& Danielopol (D.L.). 1987. - L'origine de la faune aquatique souterraine, entre le paradigme du refuge et le modèle de la colonisation active. Stygologia, 3 (4) : 345-372.

Souchon (Y.), Trocherie (F.), Fragnoud (E.) \& Lacombe (C.). 1989. - Les modèles numériques des microhabitats des poissons : application et nouveaux développernents. Rev. Sci. Eau, 2 : 807-830.

Strayer (D.) \& Bannon-O'Donnel (E.). 1988. - Aquatic microannelids (Oligochaeta and Aphanoneura) of underground waters of southeastern New-York. Amer. Midl. Natur., 119 (2) : 327-335.

Ubell (K.). 1987. - Austauschvorgänge zwischen Fluss und Grundwasser, (Transfer of water between rivers and aquifers). Deutsche Grundwasser Modellierung 31 : Teil 1 : 119-126, H.4; Teil $2: 142-149$, H.5. 